\title{
Editorial
}

\section{Advances in Personalized Mobile Services}

\author{
Federica Cena, ${ }^{1}$ Fabio Gasparetti $\left(\mathbb{D},{ }^{2}\right.$ Damianos Gavalas $\left(\mathbb{D},{ }^{3}\right.$ Shuk Y. Ho, ${ }^{4}$ Bin Liu, ${ }^{5}$ \\ and Dingqi Yang ${ }^{6}$
}

${ }^{1}$ University of Turin, 10124 Turin, Italy

${ }^{2}$ University of Roma Tre, 00154 Rome, Italy

${ }^{3}$ University of the Aegean, Syros, Greece

${ }^{4}$ The Australian National University, Canberra, ACT 0200, Australia

${ }^{5}$ IBM Thomas J. Watson Research Center, Yorktown Heights, NY, USA

${ }^{6}$ University of Fribourg, Fribourg, Switzerland

Correspondence should be addressed to Fabio Gasparetti; gaspare@dia.uniroma3.it

Received 17 May 2018; Accepted 20 May 2018; Published 26 June 2018

Copyright $(92018$ Federica Cena et al. This is an open access article distributed under the Creative Commons Attribution License, which permits unrestricted use, distribution, and reproduction in any medium, provided the original work is properly cited.

A large variety of user interaction with products, informational sources, or more general interactive media happens through mobile devices connected to Internet. However, desktop is still important for daytime at work audiences, and smart devices (such as tablets and smartphones) dominate the time that people spend connected to the web.

Personalization is a desired functionality for applications within mobile environments. It provides means of fulfilling users' needs more effectively and efficiently and consequently increasing users' satisfaction, overtaking the traditional onesize-fits-all paradigm. It is considered a fundamental feature for both users who are receiving services and for service providers who want to target their services to each individual, but different conceptual and technical challenges must be faced to achieve adequate adaptivity.

For personalization to be successful, information about the specific users is necessary to understand real user needs. Three principal sources of data about the user are usually considered: contextual information (e.g., geolocation and presence of other people), representations of user specific attributes, such as interests and needs, and social signals.

The motivation behind this special issue is to solicit cutting-edge research relevant to personalized mobile services, with significant advances, carry out innovative explorations, and establish foundations for further research. The special issue has attracted 12 submissions. Following a rigorous review process (including a second review round), 5 outstanding papers (acceptance rate $41 \%$ ) have been finally selected for inclusion in the special issue. The accepted papers cover a wide range of research subjects in the broader area of mobile services, such as recommender systems, routing protocols, task classification, user authentication, and notification systems.

The paper "Mobile Personalized Service Recommender Model Based on Sentiment Analysis and Privacy Concern" by Liang Xiao, Feipeng Guo, and Qibei Lu proposes a recommender model for mobile services based on the sentiment analysis, taking into account privacy considerations. The article, firstly, introduces a sentiment analysis algorithm based on sentiment vocabulary ontology and then clusters the users based on sentiment tendency. Then, a measurement algorithm is proposed, which integrates personality traits with privacy preference intensity, and then clusters the users based on personality traits. Last, hybrid collaborative filtering recommendation is performed by combining sentiment analysis with privacy concerns. The effectiveness of the proposed method is validated through a series of experiments using practical application datasets.

The paper "Automatic Task Classification via Support Vector Machine and Crowdsourcing" by Hyungsik Shin and Jeongyeup Paek investigates a task classification problem in personal assistant systems and proposes an automatic task classification approach. More precisely, by collecting a dataset of task commands using crowdsourcing via Amazon Mechanical Turk, they use SVM to classify a task command into one of the 32 predefined task types. In 
addition, an architecture of integrating the proposed approach with personal assistant systems is also suggested.

The paper "EpSoc: Social-Based Epidemic-Based Routing Protocol in Opportunistic Mobile Social Network" by Halikul Lenando and Mohamad Alrfaay introduces an epidemic routing technique that takes advantage of specific characteristics extracted from social networks. Epidemic routing is a flooding-based approach in which nodes continuously replicate and transmit messages to known contacts that do not already receive a copy of the message. This novel approach shows advantages in terms of the delivery ratio and latency on a real-world dataset, which includes traces of Bluetooth sightings by groups of users carrying mobile devices.

Sandeep Gupta, Attaullah Buriro, and Bruno Crispo in their paper "Demystifying Authentication Concepts in Smartphones: Ways and Types to Secure Access" provide an extensive descriptive survey on security and privacy threats to the user's personal data stored in mobile devices, with particular relevance to user authentication. The survey covers also important usability issues related to the different techniques in the literature, which may influence the decision of the adoption of one specific approach in large-scale scenarios.

Push notifications provide convenience and value to mobile app users. For instance, users can receive sports scores and news right on their lock screen and utility messages like traffic, weather, and ski snow reports. But notifications may also be considered a big distraction to users during their everyday activities. Jemin Lee et al. in their paper "Reducing Smartwatch Users' Distraction with Convolutional Neural Network" propose an AI-based notification management system for smartphone and smartwatch users based on Convolutional Neural Networks. By analyzing of a large real-world corpus, the authors prove the efficacy of the approach in the scenario of a binary classification of each notification into wanted/unwanted classes.

We do hope that this special issue will be of considerable interest to the MIS audience, highlighting state-of-the-art trends, methodologies, and applications in personalized mobile services.

\section{Acknowledgments}

We would like to sincerely thank the authors of all the submitted papers for considering our special issue and the MIS as a potential publication venue for their research results. We would also like to especially thank the authors of the accepted papers for their effort in revising and improving their work-occasionally, several times-in response to reviewers' comments. In addition, we would like to thank the anonymous reviewers for doing an excellent job in reviewing the submitted papers and making this special issue possible. Last but not least, we take this opportunity for thanking the journal $\mathrm{EiC}$ and the Editorial Board for giving us the opportunity to organize this special issue, which we sincerely believe provides a fresh, relevant, and useful overview of ongoing research in the multifaceted area of smart cities.
Federica Cena

Fabio Gasparetti

Damianos Gavalas

Shuk Y. Ho

Bin Liu

Dingqi Yang 


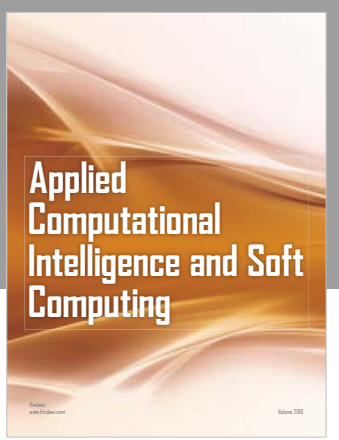

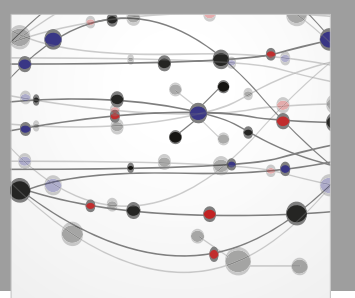

The Scientific World Journal
Submit your manuscripts at

Computing
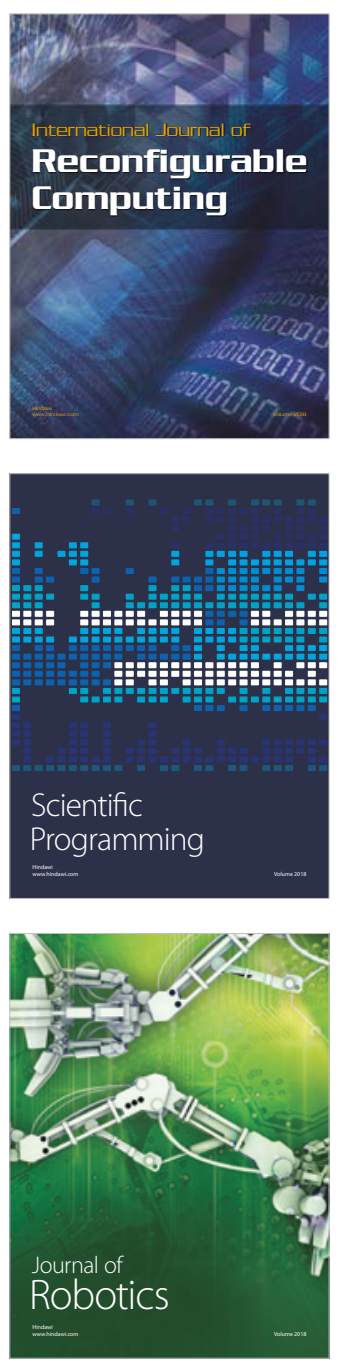

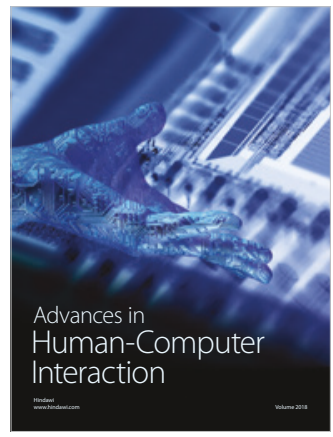

Human-Compute

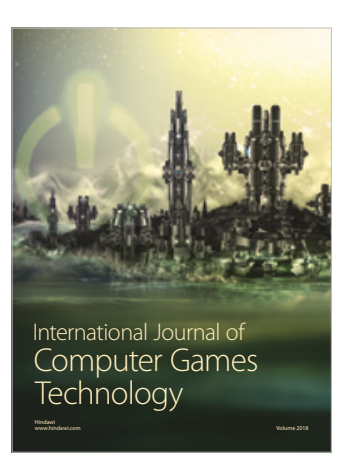

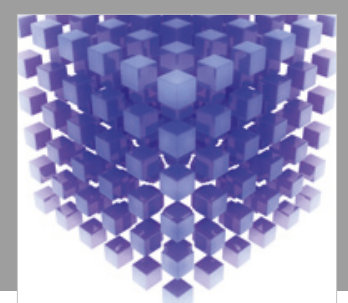

Mathematical Problems in Engineering

\section{Engincering}
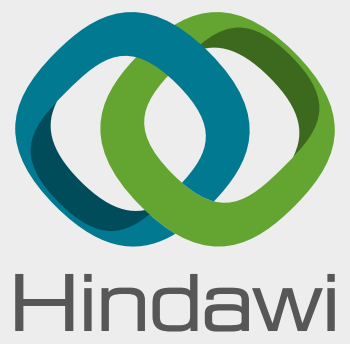

www.hindawi.com
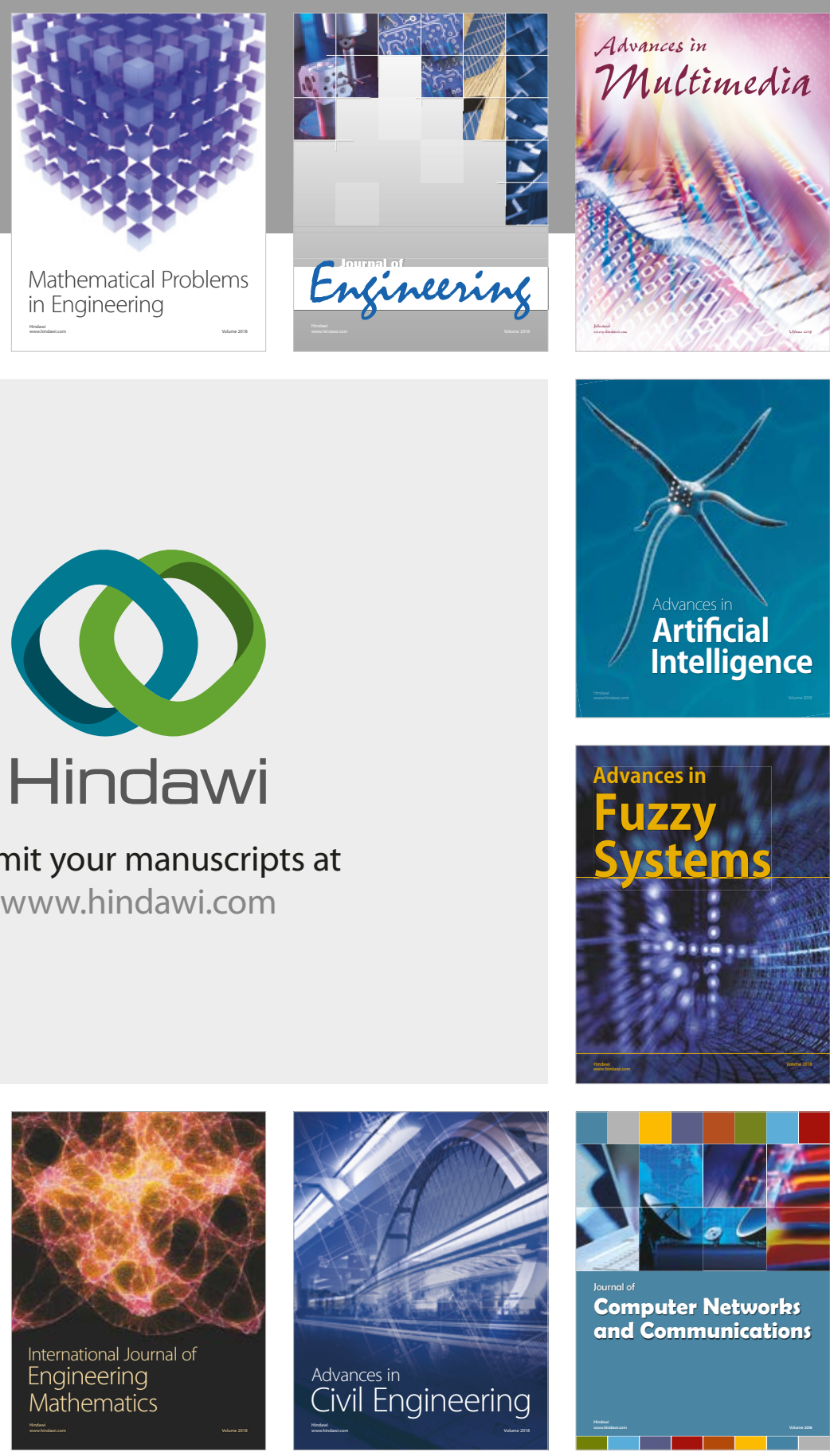

Computer Networks and Communications

Multimedia
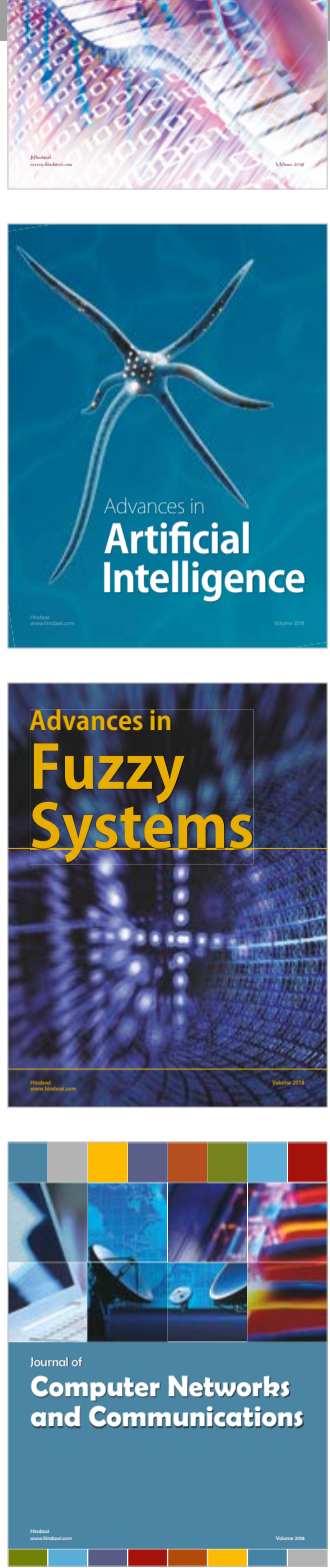

Advances in

Modelling \&

Simulation

in Engineering

interaction

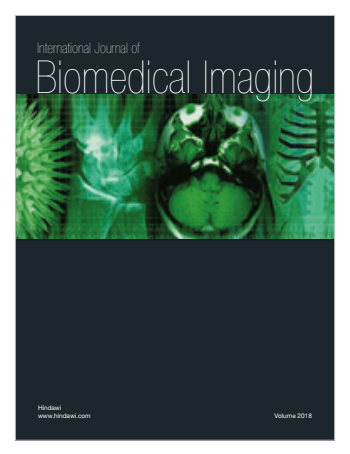

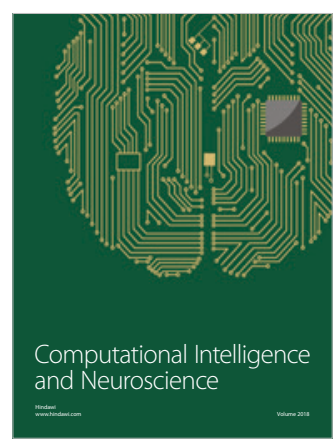

